\title{
Experimental Observation of Structural Crossover in Binary Mixtures of Colloidal Hard Spheres
}

\author{
Jörg Baumgartl, ${ }^{1, *}$ Roel P. A. Dullens, ${ }^{1}$ Marjolein Dijkstra, ${ }^{2}$ Roland Roth, ${ }^{3}$ and Clemens Bechinger ${ }^{1}$ \\ 12. Physikalisches Institut, Universität Stuttgart, 70550 Stuttgart, Germany \\ ${ }^{2}$ Soft Condensed Matter Group, Utrecht University, 3584 CC Utrecht, The Netherlands \\ ${ }^{3}$ Max-Planck-Institut für Metallforschung, 70569 Stuttgart, Germany \\ and Institut für Theoretische und Angewandte Physik, Universität Stuttgart, 70569 Stuttgart, Germany
}

(Received 13 February 2007; published 9 May 2007)

\begin{abstract}
Using confocal microscopy, we investigate the structure of binary mixtures of colloidal hard spheres with size ratio $q=0.61$. As a function of the packing fraction of the two particle species, we observe a marked change of the dominant wavelength in the pair-correlation function. This behavior is in excellent agreement with a recently predicted structural crossover in such mixtures. In addition, the repercussions of structural crossover on the real-space structure of a binary fluid are analyzed. We suggest a relation between crossover and the lateral extension of networks containing only equally-sized particles that are connected by nearest-neighbor bonds. This is supported by Monte Carlo simulations which are performed at different packing fractions and size ratios.
\end{abstract}

DOI: $10.1103 /$ PhysRevLett.98.198303

PACS numbers: 82.70.Dd, 61.20.-p

Most systems in nature and technology are mixtures of differently sized particles. Each distinct particle size introduces another length scale, and its competition gives rise to an exceedingly rich phenomenology in comparison with single-component systems. Already, the simplest conceivable multicomponent system, i.e., a binary mixture of hard spheres, exhibits interesting and complex behavior. Just a few examples include entropy driven formation of binary crystals [1-3], frustrated crystal growth [4], the Brazil nut effect [5], glass-formation [6,7], and entropic selectivity in external fields [8]. Although interaction potentials in atomic systems are more complex than those of hard spheres, the principle of volume exclusion is ubiquitous and thus always dominates the short-range order in liquids [9]. Accordingly, hard spheres form one of the most important and successful model systems in describing fundamental properties of fluids and solids. It has been demonstrated that many of their features can be directly transferred to atomic systems where fundamental mechanisms are often obstructed by additional material specific effects [10]. Binary hard-sphere systems are fully characterized by their size ratio $q=\sigma_{S} / \sigma_{B}$ with $\sigma_{i}$ the diameters of the small $(S)$ and big $(B)$ spheres and the small and big sphere packing fractions $\eta_{S}, \eta_{B}$, respectively.

The pair-correlation functions, $g_{i j}(r)$, are the central measure of structure in fluids; they describe the probability of finding a particle of size $i$ at distance $r$ from another particle of size $j$. It is well known that all pair-correlation functions in any (isotropic) fluid mixture with short-ranged interactions (not just hard spheres) exhibit the same type of asymptotic decay, which can be either purely (monotonic) exponential or exponentially damped oscillatory [11] (and references therein). This prediction, which is valid in all dimensions [12] as well as for anisotropic pair interactions [13], suggests that all pair-correlation functions decay with a common wavelength and decay length in the asymptotic regime $r \rightarrow \infty$. For binary hard-sphere mixtures where $\eta_{B} \gg \eta_{S}$ or $\eta_{S} \gg \eta_{B}$, this is rather obvious since the system is dominated by either big or small particles. The pair-correlation functions will asymptotically oscillate with a wavelength determined either by $\sigma_{B}\left(\eta_{B} \gg \eta_{S}\right)$ or $\sigma_{S}\left(\eta_{S} \gg \eta_{B}\right)$. Rather surprising is that the above statement is also valid for all other relative packing fractions where the system is not dominated by particles of a single size $[11,12]$. Accordingly, the $\left(\eta_{S}, \eta_{B}\right)$ phase diagram is divided by a sharp crossover line where the decay lengths of the contributions to $g_{i j}(r)$ with the two wavelengths become identical. Below and above this line, however, the asymptotic oscillation of the pair-correlation function is either determined by the diameter of the small spheres or that of the big spheres [14]. For long-ranged interparticle interactions, one would expect similar behavior at intermediate separations [15], but no true structural crossover in the asymptotic regime.

Despite the generic character of structural crossover and the close relationship between structural and mechanical properties, this effect has not been observed in experiments as the asymptotic behavior is difficult to observe in scattering experiments on atomic and molecular liquids. However, recent calculations suggest that structural crossover is already detectable at relatively small distances [12]. Because colloidal particles are directly accessible in real space, such systems provide an opportunity to explore the structure of binary fluids and to investigate structural crossover experimentally.

As colloidal suspension, we used an aqueous binary mixture of small melamin particles $\left(\sigma_{S}=2.9 \mu \mathrm{m}\right)$ and big polystyrene spheres $\left(\sigma_{B}=4.8 \mu \mathrm{m}\right)$. Addition of salt screens residual electrostatic interactions thus leading to an effective hard-sphere system. Since melamin has a higher mass density $\left(1.51 \mathrm{~g} / \mathrm{cm}^{3}\right)$ than polystyrene $\left(1.05 \mathrm{~g} / \mathrm{cm}^{3}\right)$, the sedimentation velocities are similar and, hence, we 
obtain a homogeneous system after mixing. The suspension was contained in a cylindrical sample cell with a silica bottom plate to allow optical imaging with an inverted confocal microscope in reflection mode (Leica TCS SP2). From the images, particle positions were obtained as described in Ref. [16]. Strong layering at the bottom wall allowed us to image only the first two-dimensional bottom layer of the three-dimensional system. We define the packing fraction as $\eta_{i}=\pi \sigma_{i}^{2} \rho_{i} / 4$, with $\rho_{i}$ the number density of component $i$. Variation of the relative packing fractions of the particles was achieved by addition of small particles to a suspension of big spheres (Fig. 1). Thus, the total packing fraction in the two-dimensional bottom layer remains constant for all samples: $\eta=0.72$. In the following, we will refer to the different samples by the sample numbers (No.) as given in Fig. 1.

Typical snapshots of the system for different packing fractions of big and small particles are shown in Figs. 2(a)2(c). The images demonstrate how the structure of the bottom layer changes from being rich in big particles (No. 1, Fig. 2(a)) to being rich in small particles (No. 10, Fig. 2(c)). Figure 2(b) (No. 5) corresponds to about the same number density of small and big spheres. In order to analyze the samples for a possible structural crossover, we calculated the pair-correlation function from the determined particle positions. To minimize statistical noise, we did not distinguish between big and small spheres. This is justified because the crossover has been predicted to be visible in all pair-correlation functions and thus also in any linear combination $[11,12]$. The dominating wavelength in the oscillations is identified by computing the total correlation function $h_{\text {tot }}(r)=\sum_{i, j} x_{i} x_{j} h_{i j}(r)=$ $\sum_{i j} x_{i} x_{j}\left[g_{i j}(r)-1\right]$, with the mole fraction $x_{i}=\rho_{i} / \sum_{i} \rho_{i}$ of component $i$ [12]. Figure 2(d) exemplarily shows

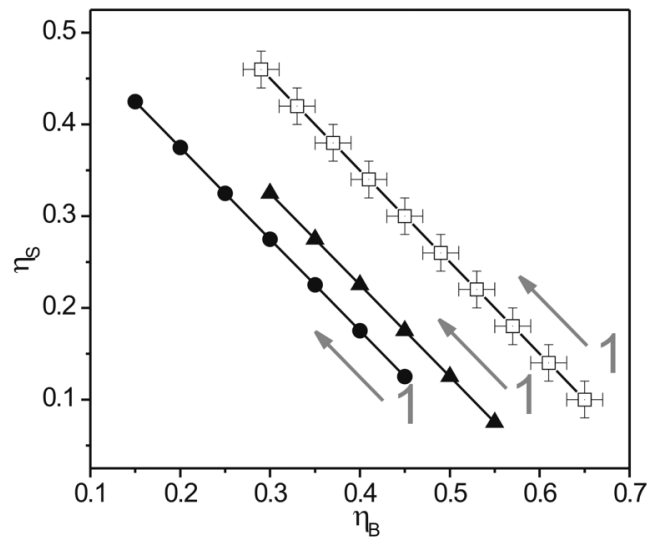

FIG. 1. Different paths with constant total packing fraction $\eta=\eta_{S}+\eta_{B}$ in the $\left(\eta_{S}, \eta_{B}\right)$-plane. Experimental data (open symbols: $\eta=0.72, q=0.61$ ) are sorted into ten bins. The bin size is indicated by the "error bars." Closed symbols correspond to the MC-simulations (solid triangle: $\eta=0.62, q=0.4$ ) and (solid circle: $\eta=0.57, q=0.5$ ). For convenience, all samples are labeled with numbers increasing in the direction indicated by the arrows. $\ln \left|h_{\text {tot }}(r)\right|$ for samples No. 1, 5, and 9. Note that in this representation, the oscillation wavelength is halved. The correlation functions of samples No. 1 and 9 clearly oscillate with a single wavelength, respectively, given by $\approx \sigma_{B} / 2$ and $\approx \sigma_{S} / 2$. In contrast, sample 5 does not show a dominating wavelength but an interference of different length scales which is typical near the structural crossover. It is important to mention that this intermediate behavior is only observed for samples Nos. 5 and 6, i.e., only for about $10 \%$ of the entire range over which $\eta_{B}$ and $\eta_{S}$ was varied. The experimentally identified crossover region is in excellent agreement with the theoretically calculated value of $\eta_{S} \approx 0.3$ at those size ratios, which were determined from the decay of the pair-correlation
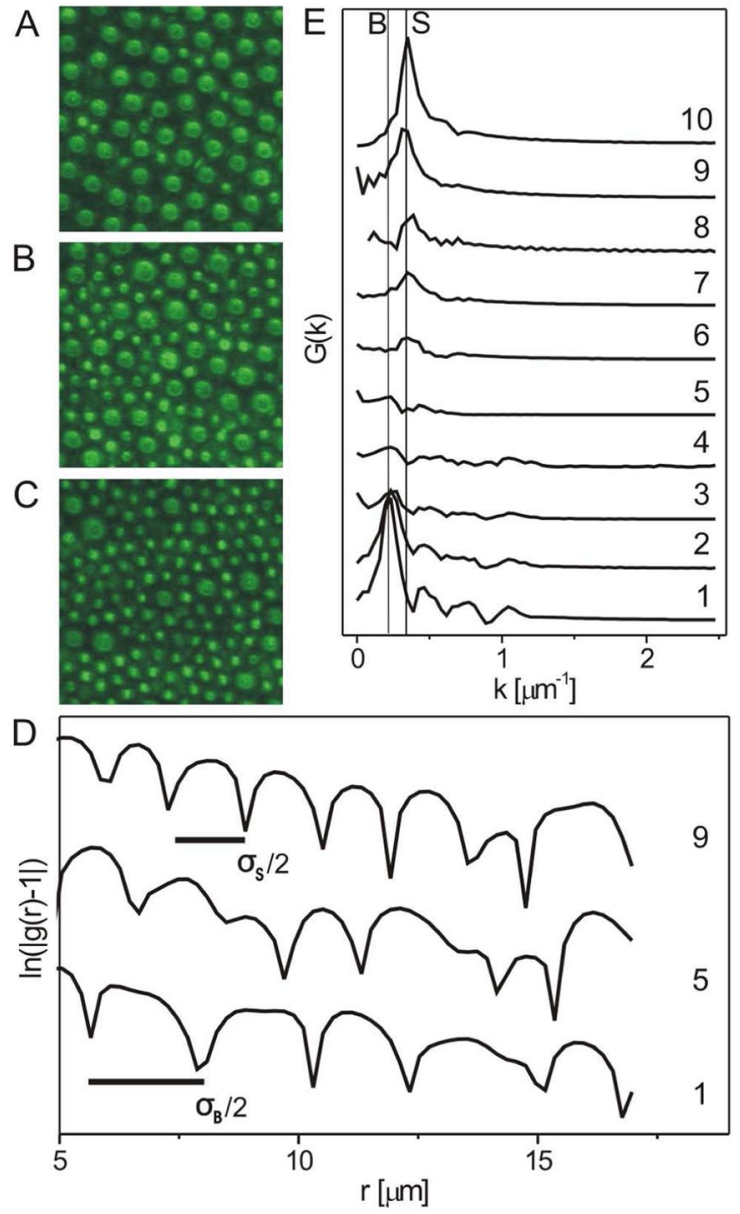

FIG. 2 (color online). (a-c) Typical snapshots of the bottom layer of a binary mixture observed with a confocal microscope used in reflection mode. The mixtures correspond to sample 1 (a), 5 (b), and 10 (c). The field of view is $40 \times 40 \mu \mathrm{m}^{2}$. (d) Logarithmic plot of the total correlation functions $h_{\text {tot }}(r)$ for the experimental binary mixtures with $\eta=0.72 \pm 0.04$. Correlation functions are plotted for sample numbers 1, 5 and 9 (compare Fig. 1) and are shifted in vertical direction for clarity. The horizontal bars correspond to $\sigma_{B} / 2$ and $\sigma_{S} / 2$, respectively. (e) Fourier-transforms of $h_{\text {tot }}(r)$ for the experimental data points (compare Fig. 1). Vertical lines indicate the wave vectors $k$ corresponding to the diameters of the small $(S)$ and big particles $(B)$, respectively. 
functions calculated within density functional theory in the test particle limit [17]. Figure 2(e) shows the Fourier transforms of $h_{\text {tot }}(r)$ for all samples where the rather sudden change of the dominating wavelength is seen more clearly [18]. At small and high packing fractions, the correlations are clearly dominated by frequencies corresponding to either small or large particles (vertical lines) while around sample No. 5, hardly any dominating frequency is observed. This experimentally confirms structural crossover as well as its occurrence at intermediate particle distances.

So far, structural crossover has been discussed in terms of pair-correlation functions, i.e., spatially averaged quantities. Since our experiments naturally provide detailed structural information, we investigate what the repercussions are of the structural crossover on the instantaneous real-space structure. We first subjected a Delaunay triangulation, which can easily be generalized to three dimensions, to the set of particle centers and identified nearestneighbor bonds between big-big (black), big-small (yellow), and small-small (red) particles, respectively (see Fig. 3). As observed in Fig. 3, sample 1 predominantly consists of big-big bonds which form a large network spreading across the entire field of view. With increasing sample No., i.e., increasing $\eta_{S}$, the number of small-small bonds increases, which leads to fragmentation of the bigbig network into smaller, randomly distributed patches. At large sample numbers, the role of big and small particles is inverted and small-small bonds form a network spanning the entire area (No. 10). Having distinguished between different bond-types, a natural and well-known measure of the spatial extend of a network formed by $n^{i}$ particles of size $i$ at positions $\vec{x}_{k}^{i}\left(k=1, \ldots, n^{i}\right)$ is given by the radius of gyration $R_{g}^{i}=\sqrt{\frac{1}{n^{i}} \sum_{k=1}^{n^{i}}\left(\vec{x}_{k}^{i}-\vec{R}_{0}^{i}\right)^{2}}$, with $\vec{R}_{0}^{i}$ the centroid position of the network. Computing this quantity for all, say $N_{C}^{i}$, networks formed by connected particles of size finally yields a weighted averaged radius of gyration $\left\langle R_{g}^{i}\right\rangle=\frac{1}{N^{i}} \sum_{m=1}^{N_{C}^{i}} n_{i}(m) R_{g}^{i}(m)$ where $N^{i}$ denotes the total number of particles $i$. We calculated $\left\langle R_{g}^{i}\right\rangle$ for networks consisting of connected big or small particles and plotted these values for our experimental data in Fig. 4(a) as a function of the sample number. At small and high sample numbers, the quantities saturate while a relatively sharp transition with an intersection point occurs around sample 6. This location is indeed in very good agreement with the crossover transition as determined from the correlation functions in Fig. 2 and density functional theory (also indicated in Fig. 4(a)). This suggests that the structural crossover corresponds to a competition between the sizes of networks consisting of connected big or small particles, respectively.

As structural crossover is also predicted for other size ratios and packing fractions, we use Monte Carlo (MC) simulations to test our findings for more dilute systems with size ratios $q=0.5(\eta=0.57)$ and $q=0.4(\eta=$ 0.62 ). The corresponding paths through the phase diagram (see closed symbols in Fig. 1) were obtained from 2dimensional simulations with a fixed number of particles of about $0<N<3000$ for both species and box areas of about $1500 \sigma_{B}^{2}$ employing periodic boundary conditions. From the configurational snapshots, we first determined the region of crossover by analyzing $h_{\text {tot }}(r)$ (the correlation functions are sampled using $10^{4} \mathrm{MC}$ cycles per particle). Then, we performed the above described Delaunay triangulation to calculate $\left\langle R_{g}^{i}\right\rangle$ for networks of connected big or small particles, respectively. The corresponding radii of gyration are plotted in Figs. 4(b) and 4(c) and show a similar behavior as in the experiment. Again, the intersection points are consistent with the crossover region as
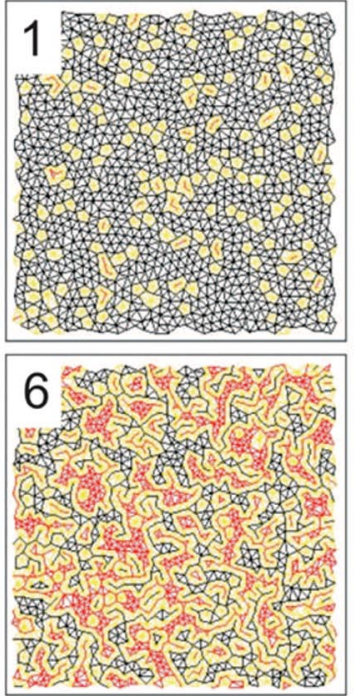
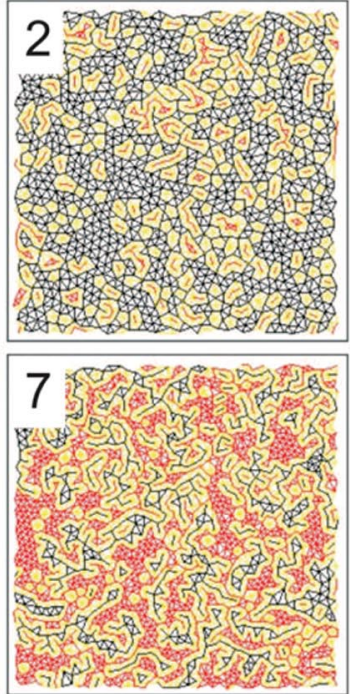
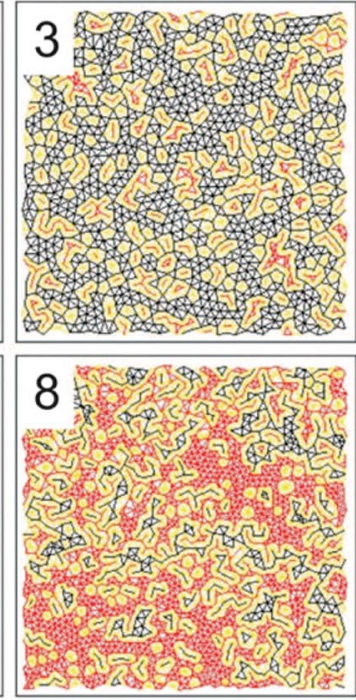
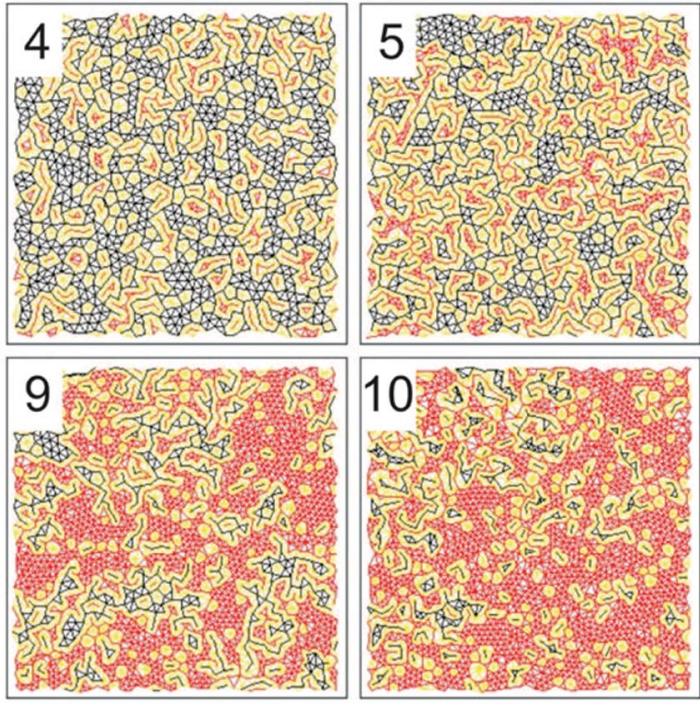

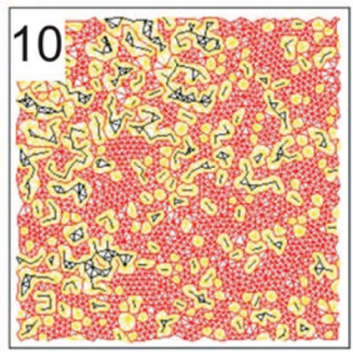

FIG. 3 (color). Visualization of the different bond-types as determined by a Delaunay triangulation: big-big (black), big-small (yellow), and small-small (red). Different plots correspond to the sample numbers as indicated in Fig. 1 . The field of view is $180 \times$ $180 \mu \mathrm{m}^{2}$. 
$\eta_{s}$

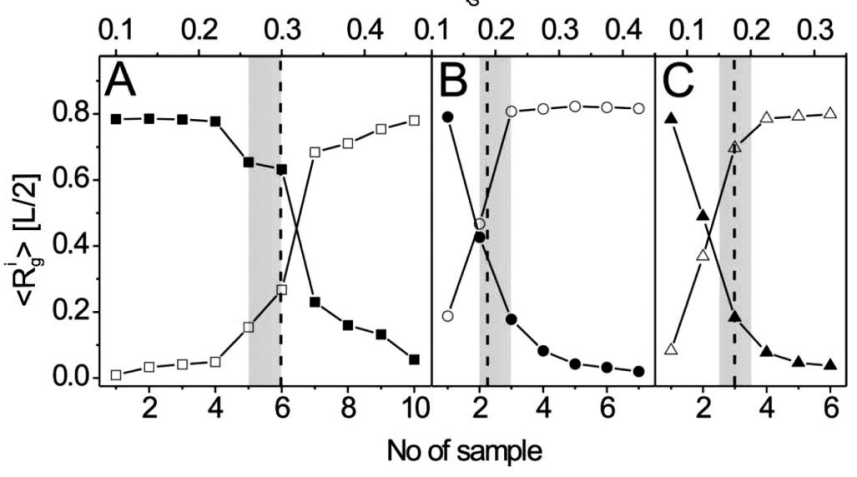

structural crossover is a generic feature of mixtures with competing length scales. Moreover, it shows interesting similarities with force chains in granular matter [19] and glassy systems $[6,7,20]$ of dissimilar sized particles. Therefore, our finding may help to gain more insight into structure-related properties in binary systems at an universal level.

We thank Bob Evans for stimulating discussions and helpful comments during preparation of the manuscript. R. P. A. D. acknowledges the Alexander von Humboldt Foundation for financial support.

FIG. 4. Averaged radii of gyration $\left\langle R_{g}^{i}\right\rangle$ (normalized to $L / 2$ with $L^{2}$ the field of view) of networks formed by large (solid symbols) and small particles (open symbols) as a function of the sample number for (a) the experimental data, (b) the MCsimulations at $\eta=0.57$ and $q=0.5$ and, (c) the MCsimulations at $\eta=0.62$ and $q=0.4$. The corresponding packing fraction of small particles $\eta_{S}$ is indicated as well. The gray area and the dashed line, respectively, indicate the crossover as inferred from the correlation functions and from density functional theory.

inferred from the correlation functions and DFT calculations. Note that the crossover region sensitively depends on the size ratio and packing fractions. Both the experiment and Monte Carlo simulations show that structural crossover is accompanied by a pronounced change in the typical size of networks consisting of connected big and small particles. By introducing small particles into a system of big spheres, connections between big particles are broken and, at the same time, connections between small particles are made. Additional calculations suggest that the system segregates into networks consisting either of big or small particles due to the competition between the free volume and configurational entropy. For our system parameters, this effect is too weak to drive a phase separation but has a profound influence on the real-space structure of the system. This sensitively affects the typical size of networks containing connected, equally-sized particles and thereby the chance of finding another particle with the same size at a relatively large distance. Consequently, the change from $\left.\left\langle R_{g}^{B}\right\rangle\right\rangle\left\langle R_{g}^{S}\right\rangle$ to $\left\langle R_{g}^{S}\right\rangle>\left\langle R_{g}^{B}\right\rangle$ (and vice versa) provides a simple real-space argument why the oscillation wavelength of the asymptotic behavior of $g_{i j}(r)$ is either set by $\sigma_{B}$ or $\sigma_{S}$.

We have experimentally demonstrated the structural crossover in a binary colloidal hard-sphere system. Furthermore, we show that structural crossover is strongly coupled to the size of networks containing connected equally-sized particles only. Going across the structural crossover, the size ratio of such networks comprised by either connected big or small particles is reversed. We believe this real-space configurational picture of structural crossover is not just applicable to binary hard spheres, as

*Electronic address: j.baumgartl@ physik.uni-stuttgart.de

[1] P. Bartlett, R. H. Ottewill, and P. N. Pusey, Phys. Rev. Lett. 68, 3801 (1992).

[2] A. B. Schofield, Phys. Rev. E 64, 051403 (2001).

[3] M.D. Eldrige, P. A. Madden, and D. Frenkel, Nature (London) 365, 35 (1993).

[4] V. W. A. de Villeneuve, R. P. A. Dullens, D. G. A. L. Aarts, E. Groeneveld, J. H. Scherff, W. K. Kegel, and H. N. W. Lekkerkerker, Science 309, 1231 (2005).

[5] D. C. Hong, P. V. Quinn, and S. Luding, Phys. Rev. Lett. 86, 3423 (2001).

[6] T. Eckert and E. Bartsch, Phys. Rev. Lett. 89, 125701 (2002).

[7] D. N. Perera and P. Harrowell, Phys. Rev. E 59, 5721 (1999).

[8] R. Roth and D. Gillespie, Phys. Rev. Lett. 95, 247801 (2005).

[9] S. Sastry, T. M. Truskett, P. G. Debenedetti, S. Torquato, and F. H. Stillinger, Mol. Phys. 95, 289 (1998).

[10] W. Poon, P. Pusey, and H. N. W. Lekkerkerker, Phys. World 9, 27 (1996).

[11] C. Grodon, M. Dijkstra, R. Evans, and R. Roth, J. Chem. Phys. 121, 7869 (2004).

[12] C. Grodon, M. Dijkstra, R. Evans, and R. Roth, Mol. Phys. 103, 3009 (2005).

[13] S. V. Savenko and M. Dijkstra, Phys. Rev. E 72, 021202 (2005).

[14] For very asymmetric size ratios, i.e., $q<0.3$, there can be additional regions in which oscillations at intermediate wavelength can be observed.

[15] R. J. F. L. de Carvalho, R. Evans, D. C. Hoyle, and J. R. Henderson, J. Phys. Condens. Matter 6, 9275 (1994).

[16] J. C. Crocker and D. G. Grier, J. Colloid Interface Sci. 179, 298 (1996).

[17] R. Roth, R. Evans, and S. Dietrich, Phys. Rev. E 62, 5360 (2000).

[18] In two dimensions for radial symmetric functions, the Fourier transform becomes a Besseltrans form. However, for the identification of the dominant wavelength, the usual Fourier transform, which is numerically easier to handle, predicts equivalent results.

[19] C. S. O’Hern, S. A. Langer, A. J. Liu, and S. R. Nagel, Phys. Rev. Lett. 86, 111 (2001).

[20] N. Hoffmann, F. Ebert, C. N. Likos, H. Löwen, and G. Maret, Phys. Rev. Lett. 97, 078301 (2006). 